\title{
Effects of Varicella Vaccine Time of First Dose and Coverage of Second Dose - Beijing and Ningbo, China, 2012-2018
}

\author{
Yuewei Chen'; Rui Ma'; Yunyan Zhang ${ }^{3}$; Xudong $\mathrm{Li}^{1}$; Dapeng Yin ${ }^{1, *}$
}

\section{Summary \\ What is already known about this topic? \\ The effectiveness of the two-dose vaccination schedule of varicella is better than that of one dose, but the vaccination schedule and coverage of varicella varies based on provinces in the mainland of China and has differing effects. \\ What is added by this report? \\ Earlier vaccination of the first dose may reduce the varicella incidence, and improving the vaccination coverage rates of the second dose will further reduce the varicella incidence. \\ What are the implications for public health practice? \\ Taking the first dose of vaccination promptly at 12 months old and improving the coverage of second dose of vaccination may play an important role in varicella prevention and control in China.}

Varicella-zoster virus (VZV) is a highly contagious herpesvirus that can cause varicella (chickenpox) in children and herpes zoster (shingles) in adults, and most people would become infected by mid-adulthood in the absence of a varicella vaccination program (1). Epidemiological data on varicella is needed before the vaccine can be included into China's Expanded Program on Immunization (EPI).

The timing of administering the vaccination is crucial for controlling the spread of varicella, but the mainland of China lacks a uniform schedule for these vaccinations. This study compared Beijing and Ningbo of Zhejiang Province to analyze the differences in varicella incidence when compared to different vaccination schedules for the first dose and different coverage of the second dose.

After 2007, Beijing and Ningbo have required the compulsory reporting of varicella incidence through the National Notifiable Diseases Reporting System (NNDRS) of the mainland of China. The epidemiological data from regions where compulsory reporting had been implemented provide some valuable information, and all the data in this paper is from the NNDRS (2007-2018).

Beijing and Ningbo are developed areas in the mainland of China. The immunization program and infectious disease surveillance of the two cities are well organized and their data on varicella is comparatively higher quality within China (2-4). A two dose immunization schedule is recommended in the two cities with little difference for the first dose. The first dose of vaccine was set to be administered at 18 months of age in Beijing starting in 2007 and 12-18 months in Ningbo starting from 2012. The second dose was set to be administered at 4 years of age in Beijing since 2012 and Ningbo since 2014. The varicella inoculation rate for the first dose is higher than the second dose and the inoculation rate for Beijing is higher than Ningbo. (Figure 1)

There is a noticeable difference in varicella incidence in age group 1 (12 to 24 months) in the two cities with the incidence in Beijing being much higher than that of Ningbo. In Beijing, the incidence in age group 1 initially increased and then declined slightly, but Ningbo had a relatively low incidence without significant changes. (Figure 2)

After the implementation of the two-dose schedule in Beijing from 2012, the total population incidence decreased significantly but increased slightly after 2016. Ningbo also showed decreases after introducing the second dose of vaccine in 2014 but had dramatic increases after 2016.

Further analysis of the incidence of the sub-age group 0 years old to 15 years old in Ningbo reveals a prominent increase for the age group of 9 years old to 10 years old (from 9 years old to 14 years old) after 2014 (Figure 2), and the peak incidence of Ningbo is backward from 4-8 years old in 2012, to 9-13 years old in 2018 (Figure 3).

\section{DISCUSSION}

Most population-based data on the epidemiology of varicella are from high-income countries, data from 


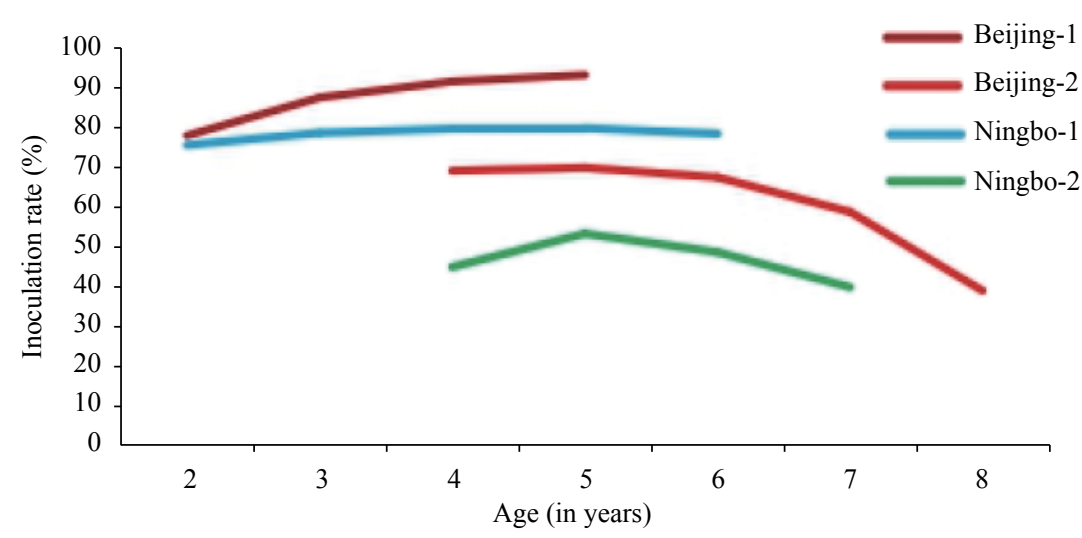

FIGURE 1. The inoculation rate of different dose of varicella vaccines in Beijing and Ningbo city, China, 2017. Beijing-1 and Ningbo-1 is the inoculation rate of the first dose of varicella vaccines in Beijng and Ningbo, Beijing-2 and Ningbo-2 is the inoculation rate of the second dose of varicella vaccines in Beijing and Ningbo.

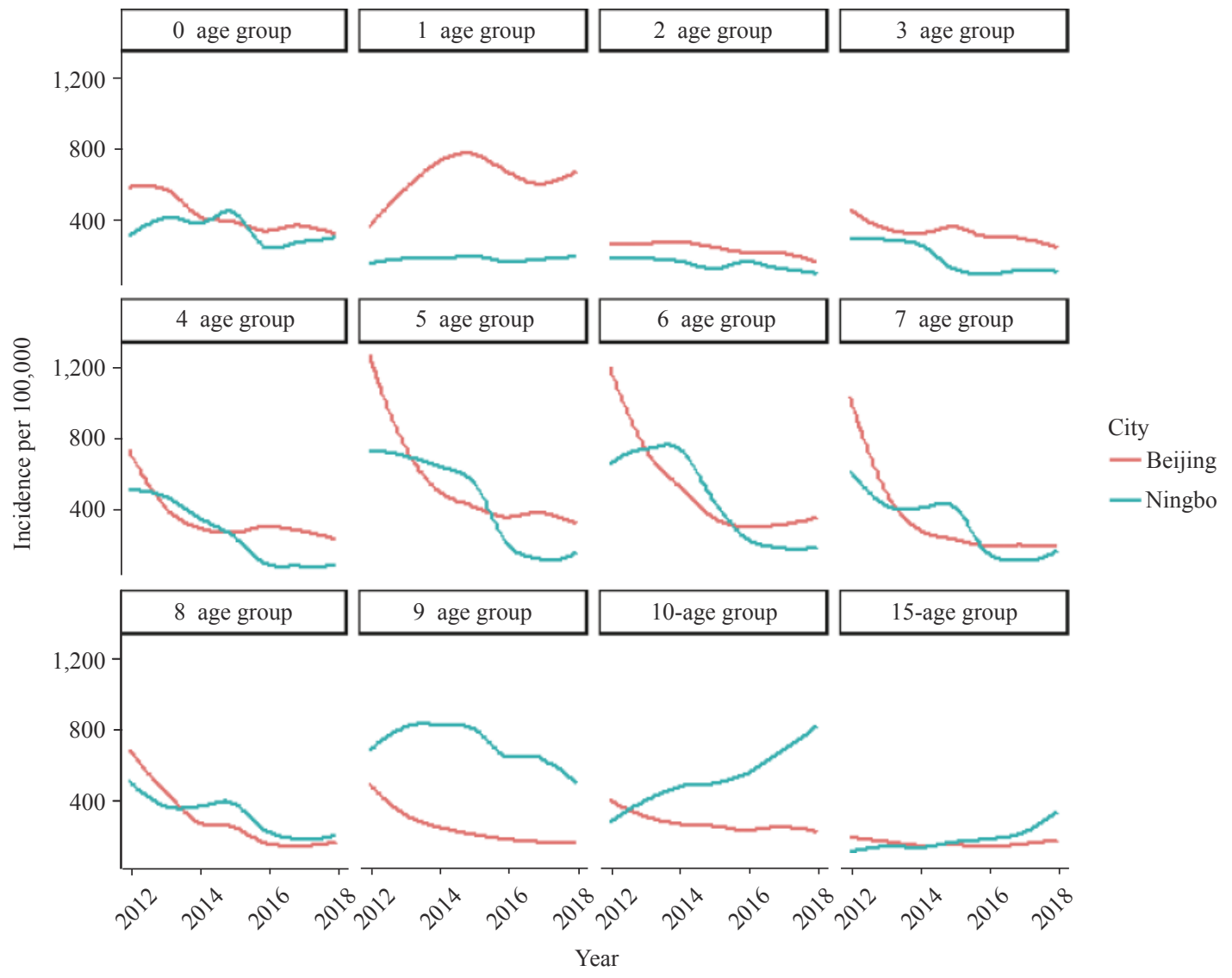

FIGURE 2. The incidence of varicella by age group from 0 to 15 after taking the vaccination in Beijing and Ningbo city, China, 2012-2018. The incidence from 0 to 9 represents each year age group. The incidence of 10 and 15 age group represents the incidence of 10-14 years and 15-19 years, respectively.

low-to-middle income countries are comparatively limited (1). The epidemiological data of Beijing and Ningbo in the mainland of China can provide some useful information for the preparation of vaccine immunization strategies in China. The epidemiological profiles of varicella in the two cities are different as are the immunization schedules, surveillance requirements, and immunization coverages.

This investigation reported a comparative study of the varicella vaccination in Beijing and Ningbo based on policies and coverage by year, and associating the policies and coverage levels with the incidence of 


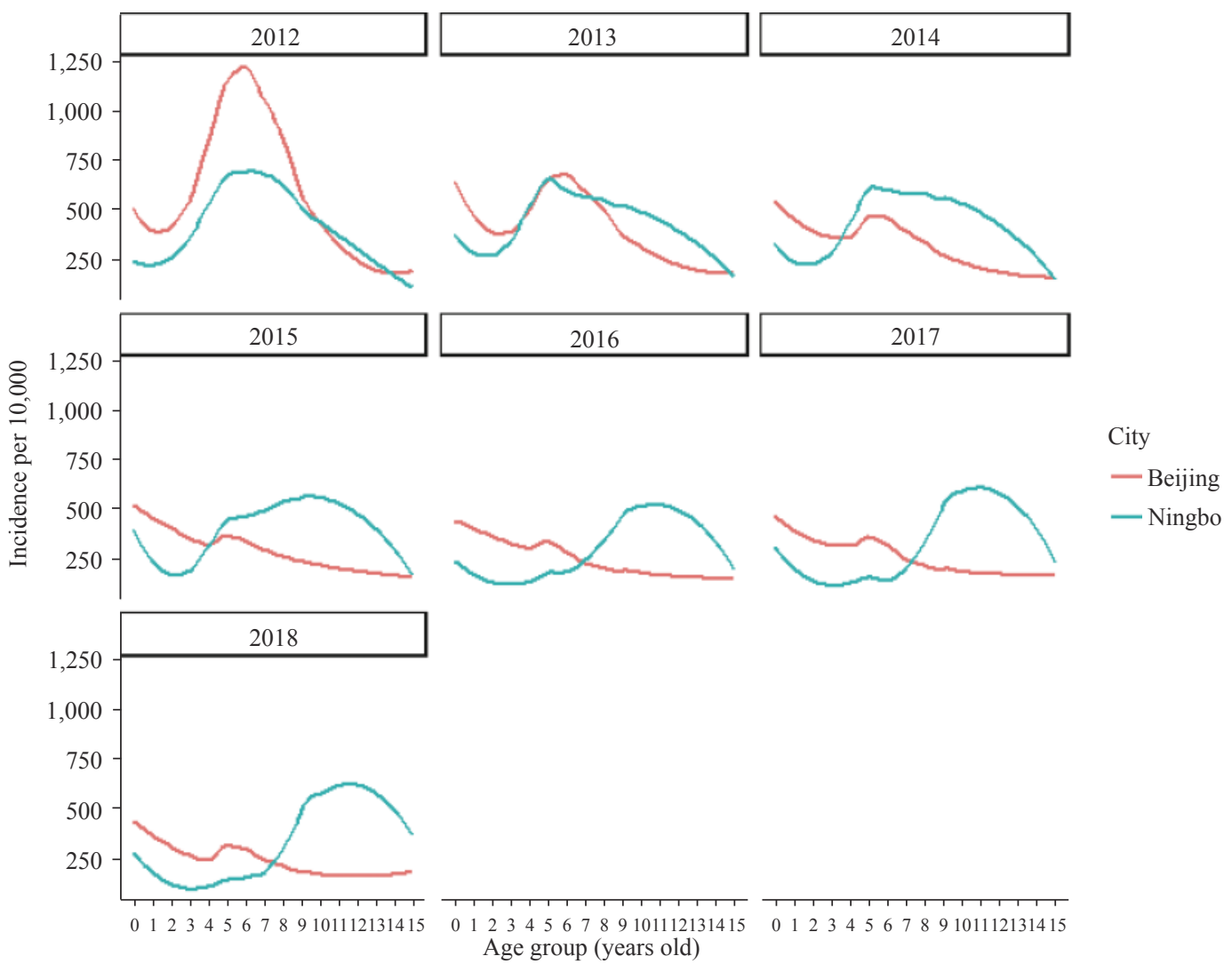

FIGURE 3. The incidence of varicella by year from 2012 to 2018 in Beijing and Ningbo city, in China from age group 0 to 15. The incidence from 0 to 14 represents each year age group. The incidence of 15 age group represents the incidence of 15-19 years, respectively.

varicella by age and by year. The research analyzed the possible reason of the higher varicella incidence of Beijing compared with that of Ningbo among age group 1 (12-24 months). The difference may not emerge from baseline data differences because the incidence in both cities is similar in age group $0(0-12$ months) (Figure 2). However, a likely reason is the difference in the time of administration of the first dose of the varicella vaccine. While the administration of the first dose is recommended at 12-18 months of age by the World Health Organization (WHO) (1), the time of the first dose in the mainland of China varies between regions. For instance, the time of vaccine administration in Beijing is set at 18 months, while Ningbo is set at $12-18$ months. The first dose of vaccination being set at the age of 18 months may cause a susceptible population of children aged 12-18 months to miss an earlier immunization.

The total incidence of varicella under 15 decreased from 2012 in Beijing and slightly increased after 2016 (2) after the two dose schedule was introduced into the local program. Similarly, after the two-dose schedule was administered after 2014 in Ningbo, the incidence decreased and increased noticeably. This may be due to the coverage of the second dose being lower in both Beijing (39.79\%-70.54\%) and Ningbo (40.63\%$54.01 \%)$. The coverage of second dose in both areas did not reach the $85 \%$ threshold that was recommended by the WHO to establish herd immunity (5). In addition, the issue of breakthrough infections exists in which a vaccinated individual becomes sick from the illness the vaccine was meant to prevent, and a high incidence of breakthrough cases has appeared in children aged 3-6 years old (6). Therefore, after a second dose of vaccine in 2012 for Beijing, there may have been some breakthrough cases after 2015.

By comparing the incidence of varicella in different age groups between Beijing and Ningbo (Figure 3), we can find that the incidence of almost every age group decreased dramatically from 2012 in Beijing. But for Ningbo, the age of peak incidence was changed from $4-8$ years old in 2012 to $9-13$ years old in 2018 . This is likely due to the vaccine coverage in Ningbo being much lower than that of Beijing, which may cause many susceptible cases to accumulate. The WHO and 
many countries have suggested that the introduction of widespread childhood varicella vaccination would decrease exposure to VZV in the population and result in an older age distribution of the remaining cases. Since complication rates in adults are higher than those in children, an increase in the peak age of infections could lead to increases in the overall morbidity even though the total number of cases would be reduced. This should be carefully monitored in Ningbo after the two-dose schedule was introduced into the local vaccine program.

This surveillance data can inform policy recommendations. The study results recommend lowering the age of first-dose vaccination in Beijing, paying attention to the increasing age of infection in Ningbo, and improving coverage of the second dose of varicella vaccine in Ningbo.

This study is subject to some limitations. First, the data was passively collected from the NNDRS and may be subject to underreporting that may lower the reporting rate of varicella. Second, this study was limited in scope to Beijing and Ningbo, so the effects of the two-dose vaccination schedule for varicella may not be generalizable to other cities or to China as a whole.

In conclusion, this paper analyzes the varicella data reported from the NNRDS. The most valuable information can be shared by local CDCs when combined with more detailed information on local varicella vaccination rates and vaccination management. As the incidence of varicella has been controlled to a relatively low level, decreasing the time of the administration of the first dose of the vaccine to 12 months of age may further decrease the incidence of varicella. Improving the coverage of second dose especially for the age 6-14 is important to control varicella in China.

doi: $10.46234 / \mathrm{ccdcw} 2020.136$

\# Corresponding author: Dapeng Yin, yindp@chinacdc.cn.

${ }^{1}$ Office of Epidemiology, Chinese Center for Disease Control and
Prevention, Beijing, China; ${ }^{2}$ Ningbo Center for Disease Control and
Prevention, Ningbo, Zhejiang, China; ${ }^{3}$ Suzhou Center for Disease
Control and Prevention, Suzhou, Jiangsu, China.

Submitted: March 12, 2020; Accepted: April 16, 2020

\section{REFERENCES}

1. Varicella and herpes zoster vaccines: WHO position paper, June 2014 https://www.who.int/wer/2014/wer8925.pdf. [2020-03-12].

2. Fu JY, Jiang C, Wang JG, Zhao FX, Ma TW, Shi RJ, et al. Epidemiology of varicella in Haidian district, Beijing, China2007-2015. Vaccine 2017;35(18):2365 - 71. http://dx.doi.org/10.1016/ j.vaccine.2017.03.044.

3. Zhang X, Yu YC, Zhang J, Huang ST, Wang ZZ, Zhang JJ, et al. The epidemiology of varicella cases among children in Beijing's Fengtai District from 2008 to 2012. Vaccine 2014;32(29):3569-72. http://dx.doi.org/10.1016/j.vaccine.2014.04.069.

4. Suo LD, Lu L, Wang QH, Yang F, Wang X, Pang XH, et al. Varicella outbreak in a highly-vaccinated school population in Beijing, China during the voluntary two-dose era. Vaccine 2017;35(34):4368- 73 . http://dx.doi.org/10.1016/j.vaccine.2017.06.065.

5. Streng A, Seeger K, Grote V, Liese JG. Varicella vaccination coverage in Bavaria (Germany) after general vaccine recommendation in 2004. Vaccine 2010;28(35):5738 - 45. http://dx.doi.org/10.1016/j.vaccine. 2010.06.007.

6. Oh SH, Choi EH, Shin SH, Kim YK, Chang JK, Choi KM, et al. Varicella and varicella vaccination in South Korea. Clin Vaccine Immunol 2014;21(5):762 - 768. http://dx.doi.org/10.1128/CVI.0064513. 